\title{
New Delhi metallo- $\beta$-lactamase- producing Acinetobacter baumannii: a novel paradigm for spreading antibiotic resistance genes
}

\author{
Rémy A Bonnin', Laurent Poirel ${ }^{1,2} \&$ Patrice Nordmann ${ }^{* 1,2}$
}

\begin{abstract}
The impact of carbapenemase production among clinically significant Gram-negative rods is becoming a major medical issue. To date, Acinetobacter baumannii has been considered as a final recipient of carbapenemase genes (imipenemase, Verona metallo- $\beta$-lactamase, Guiana extended-spectrum $\beta$-lactamase and Klebsiella pneumonia carbapenemase types) from Enterobacteriaceae and Pseudomonas aeruginosa. However, recent findings regarding the spread of the $b a_{\mathrm{NDM}}$ carbapenemase genes revealed that A. baumannii likely acts as a source of emerging antibiotic resistance genes. The analysis of genetic structure surrounding the $b / a_{\mathrm{NDM}-1}$ gene revealed that the genetic structure $(\operatorname{Tn} 125)$ responsible for its dissemination most probably originates from Acinetobacter. Moreover, analysis of the $b / a_{\mathrm{NDM}-1}$ gene itself demonstrated that it might be constructed in Acinetobacter through a recombination event with another resistance gene found in A. baumannii (aphA6). This novel paradigm highlights a novel and unexpected role played by $A$. baumannii.
\end{abstract}

Members of the genus Acinetobacter seem to have the ability to quite rapidly acquire resistance to new antibiotics [1]. The rise of resistance to broad-spectrum antibiotics such as aminoglycosides, expanded-spectrum cephalosporins, carbapenems and tigecycline in Acinetobacter baumannii has left few therapeutic options [2]. The main problem corresponds to the rise of carbapenem resistance in A. baumannii, which is almost always identified in multidrug-resistant clinical isolates [3]. Until now, A. baumannii has been considered a final reservoir of antibiotic resistance genes by acquisition of foreign DNA from different sources, particularly from other Gram-negative species. Many resistance genes have been identified in $A$. baumannii including those encoding extendedspectrum $\beta$-lactamases (PER-, Guiana extended-spectrum $\beta$-lactamase [GES] and VEB type) and aminoglycoside resistance genes [1,4]. The acquisition of those genes is often related to an integration of foreign DNA originating from other clinical species (Enterobacteriaceae and Pseudomonas aeruginosa) [1,2,4-7].

\section{Carbapenem resistance in $A$. baumannii}

Genes encoding metallo- $\beta$-lactamases of verona metallo- $\beta$-lactamase- and imipenemase types, as well as those encoding class A carbapenemases (Klebsiella pneumonia carbapenemase or GES), are other examples of acquisition of foreign resistance genes as a source of carbapenem resistance in A. baumannii [2]. Analysis of the genetic structures surrounding those carbapenemase genes reveals that they are not specific to Acinetobacter. The identified genetic structures argue for a transfer

'INSERM U914, Emerging Resistance to Antibiotics, K. Bicêtre, France

${ }^{2}$ Medical \& Molecular Microbiology Unit, Department of Medicine, Faculty of Science, University of Fribourg, rue Albert Gockel 3,

$\mathrm{CH}-1700$, Fribourg, Switzerland

*Author for correspondence: Tel.: +41 26300 9581; patrice.nordmann@unifr.ch

KEYWORDS

- $\beta$-lactamase $\bullet$ antibiotic resistance $\bullet$ carbapenemase - Gram-negative rods 
from Enterobacteriaceae and $P$. aeruginosa to A. baumannii.

The most important source of carbapenem resistance in $A$. baumannii is the production of carbapenem-hydrolyzing Ambler class D $\beta$-lactamases (CHDL) [7]. Five different subgroups of acquired CHDLs have been identified, namely oxacillinase, class D $\beta$-lactamase (OXA) -23, - 40, -58, -143 and -235 [2,7,8]. These five CHDL groups are specific to the Acinetobacter species and are not usually identified in other clinically relevant species, such as in Enterobacteriaceae and in $P$. aeruginosa [2,7]. In contrast to what is observed for other types of carbapenemase genes (those encoding the carbapenemases K. pneumonia carbapenemase, GES, verona metallo- $\beta$-lactamase and imipenemase), CHDL-encoding genes are associated with insertion sequences (IS) identified in Acinetobacter species (e.g., ISAba1). The natural progenitor of one of these carbapenemase subgroups (OXA-23) has been identified as being Acinetobacter radioresistens [9]. To date, this corresponds to one of the few examples of a resistance gene originating from an Acinetobacter species and targeting another Acinetobacter species.

\section{The case of the bla $a_{N D M}$ genes}

The bla ${ }_{\mathrm{NDM}-1}$ gene is one of the latest carbapenemase genes to have been reported. They have now been extensively reported in Enterobacteriaceae (Escherichia coli and K. pneumoniae), first from India, Pakistan and Bangladesh, and then from the rest of the world [10-12]. The occurrence of the $b l_{\mathrm{NDM}_{-1}}$ gene in the environment has also been evidenced in India, particularly among environmental Gram-negative species [13]. Sequencing of a bla ${ }_{\mathrm{NDM}-1}$-bearing plasmid, as part of the analysis of the resistosome of a multidrug-resistant $E$. coli, led to the identification of a remnant of ISAba125, located immediately upstream of the $b l a_{\mathrm{NDM}-1}$ gene [14]. The IS Aba125 element provided the -35 sequence of the hybrid promoter responsible for the expression of the bla $a_{\mathrm{NDM}-1}$ gene [14]. Interestingly, this IS element had been originally identified from an $A$. baumannii isolate (hence its name) without any physical association with the $b l a_{\mathrm{NDM}}$ gene [15]. Following this, IS Aba125 was identified in many other $A$. baumannii isolates $[6,16]$. By contrast, this IS has been identified in Enterobacteriaceae and $P$. aeruginosa only as a remnant of the $\operatorname{Tn} 125$ originating from Acinetobacter species and has never been identified alone in these species [17].
This observation suggests that $A$. baumannii is a likely reservoir of ISAba125.

While the first studies dealt with the bla ${ }_{\mathrm{NDM}}$-like genes from enterobacterial isolates, many $A$. baumannii isolates carrying the $b l a_{\mathrm{NDM}}$-like gene have been now identified (Table 1) $[2,18]$. The possible progenitor (still not identified) of the $b l a_{\mathrm{NDM}}$ gene is considered to be phylogenetically distant from $A$. baumannii owing to the guanine-cytosine content of this gene $\left(62 \%\right.$ for the $b a_{\mathrm{NDM}}$ gene vs from 38 [Acinetobacter calcoaceticus] up to $42 \%$ [Acinetobacter lwoffii] for genomes of Acinetobacter species). Likewise, the guanine-cytosine content of IS $A b a 125$ is $37 \%$ and the fact that IS Aba 125 has been identified alone (not associated with the bla $a_{\mathrm{NDM}-1}$ gene) seems to indicate the occurence of independent acquisition of $b l a_{\mathrm{NDM}-1}$ and ISAba125. Transfer of the bla $a_{\mathrm{NDM}-1}$ gene to $A$. baumannii is probably the result of a transfer from an unknown bacterial species to $A$. baumannii, with both donor and recipient probably being present concomitantly in a same environment. In $A$. baumannii, the bla ${ }_{\mathrm{NDM}-1}$ gene is part of a 10,099-bp composite transposon made of two copies of ISAba125 (Figure 1) [19]. Downstream of $b l a_{\mathrm{NDM}-1} / b l a_{\mathrm{NDM}-2}$, eight open reading frames have been identified (Figure 1). The first corresponds to the ble ${ }_{\mathrm{MBL}}$ gene, encoding a 121-amino acid-long protein conferring resistance to bleomycin (anticancer/antibiotic drug), previously found associated with bla $a_{\mathrm{NDM}-1}$ in enterobacterial isolates [20]. Then, several genes encoding putative proteins sharing similarities with genes identified from the Brevundimonas and Xanthomonas genera were identified [19]. Downstream, two genes encoding the GroES and GroEL chaperonin proteins, respectively, were identified. Finally, a gene encoding the putative transposase of an IS common region (ISCR)-like element was identified sharing 93\% protein sequence identity with ISCR19 [21]. ISCR elements are IS able to mobilize DNA fragment located at their left-hand extremity by rollingcircle transposition [22]. At the right-hand extremity of ISCR 21 and before the second copy of ISAba125 of Tn125, a truncated gene encoding a putative phospholipid acetyltransferase was identified, with the corresponding protein sequence sharing $91 \%$ amino acid identity with that of Acinetobacter junii (Figure 1) [19]. This finding indicates a likely intermediate state that may have occurred in an Acinetobacter species other than $A$. baumannii. Our hypothesis is 


\begin{tabular}{|c|c|c|c|c|c|c|c|}
\hline Isolates or species & Isolates (n) & $\begin{array}{l}\text { Acquired } \\
\text { carbapenemase }\end{array}$ & $\begin{array}{l}\text { Worldwide } \\
\text { clone }\end{array}$ & $\begin{array}{l}\text { MLST } \\
\text { analysis }^{\dagger}\end{array}$ & $\begin{array}{l}\text { Country of } \\
\text { isolation }\end{array}$ & $\begin{array}{l}\text { Probable } \\
\text { origin }\end{array}$ & Ref. \\
\hline \multicolumn{8}{|c|}{ Acinetobacter baumannii isolates } \\
\hline A. baumanii JH & 1 & NDM-1, OXA-23 & WWI & $\mathrm{ST} 1^{\mathrm{p}}$ & Switzerland & $\begin{array}{l}\text { Balkans } \\
\text { region }\end{array}$ & {$[18]$} \\
\hline $\begin{array}{l}\text { A. baumannii } \\
\text { ANC4097 }\end{array}$ & 1 & NDM-1, OXA-23 & WWI & $\mathrm{ST} 1^{\mathrm{p}}$ & Czech Republic & Egypt & {$[40]$} \\
\hline A. baumannii $161 / 07$ & 1 & NDM-1 & None & $\mathrm{ST} 25^{\mathrm{P}}$ & Germany & Serbia & [41] \\
\hline A. baumannii Slo & 1 & NDM-1 & None & $\mathrm{ST} 25^{\mathrm{P}}$ & Slovenia & $\begin{array}{l}\text { Balkans } \\
\text { region }\end{array}$ & {$[18]$} \\
\hline A. baumannii & 8 & NDM-1 & None & ST $85^{\mathrm{P}}$ & France & North Africa & {$[32]$} \\
\hline $\begin{array}{l}\text { A. baumannii } \\
\text { Ab11314 }\end{array}$ & 1 & NDM-1 & WWII & ST92 ${ }^{\mathrm{B}}$ & Belgium & Algeria & [42] \\
\hline $\begin{array}{l}\text { A. baumannii A28, } \\
\text { A32 and A36 }\end{array}$ & 3 & NDM-1, OXA-23 & N/A & $\mathrm{N} / \mathrm{A}$ & India & India & [26] \\
\hline A. baumannii & 1 & NDM-1, OXA-23 & N/A & $\mathrm{N} / \mathrm{A}$ & Japan & India & [43] \\
\hline $\begin{array}{l}\text { A. baumannii } \\
\text { ABCA207, ABC3229, } \\
\text { ABC4289, ABWA7 }\end{array}$ & 4 & NDM-1 & N/A & N/A & China & China & [27] \\
\hline A. baumannii $\mathrm{ML}$ & 1 & NDM-2 & None & ST103 ${ }^{P}$ & Germany & Egypt & [33] \\
\hline $\begin{array}{l}\text { A. baumannii I1, } 12 \text {, } \\
\mid 15,116,117\end{array}$ & 5 & NDM-2 & None & ST103 ${ }^{P}$ & Israel & $?$ & [34] \\
\hline $\begin{array}{l}\text { A. baumannii 124, } \\
132\end{array}$ & 2 & NDM-2 & None & ST103 ${ }^{P}$ & $\begin{array}{l}\text { United Arab } \\
\text { Emirates }\end{array}$ & $\begin{array}{l}\text { United Arab } \\
\text { Emirates }\end{array}$ & [35] \\
\hline \multicolumn{8}{|c|}{ Acinetobacter non-baumannii isolates } \\
\hline A. Iwoffii WJ10621 & 1 & NDM-1 & N/A & N/A & China & $?$ & [44] \\
\hline A. pittii D499 & 1 & NDM-1 & N/A & N/A & China & $?$ & {$[45]$} \\
\hline A. junii 1454 & 1 & NDM-1 & N/A & N/A & China & $?$ & [46] \\
\hline Acinetobacter spp. & 9 & NDM-1 & N/A & N/A & China & China & {$[28]$} \\
\hline A. Iwoffii SGC-HZ9 & 1 & NDM-1 & N/A & $\mathrm{N} / \mathrm{A}$ & China & China & [29] \\
\hline A. pittii & 27 & NDM-1 & N/A & ST63 ${ }^{\mathrm{P}}$ & China & China & {$[47]$} \\
\hline A. johnsonii & 2 & NDM-1 & $\mathrm{N} / \mathrm{A}$ & $\mathrm{N} / \mathrm{A}$ & China & China & {$[30]$} \\
\hline
\end{tabular}

that an Acinetobacter species acquired the $b a_{\mathrm{NDM}}$ gene from an environmental species, probably via a natural transformation process (a physiological process encoded by a wide range of bacteria, permitting the uptake of exogneous DNA via the binding of dsDNA on a specific membrane receptor followed by the entry of ssDNA; finally, homologous recombination is necessary to incorporate the exogeneous DNA) (Figure 2). In that species, the transposon $\mathrm{Tn} 125$ was built. Subsequently, $A$. baumannii acquired the bla ${ }_{\mathrm{NDM}}$ gene through the acquisition of this transposon. After its dissemination among Acinetobacter species, an interspecies transfer via a broadhost range plasmid permitted the acquisition of Tn 125 by Enterobacteriaceae or $P$. aeruginosa. This step was followed by a large dissemination of the $b l a_{\mathrm{NDM}}$ in Enterobacteriaceae (Figure 2).

Interestingly, a recent study suggested that the bla $a_{\mathrm{NDM}-1}$ has been constructed in Acinetobacter [23]. Precise genetic analysis of the blaNDM-1 gene itself revealed an interesting feature. It shared the $5^{\prime}$ end sequence of the aminoglycoside resistance gene aphA6 (displaying 100\% identity in the spacer sequence [260 bp] between ISAba125 and either the $b l a_{\mathrm{NDM}-1}$ or $a p h A 6$ genes and the first 20 nucleotides of the both genes) [23]. Figure 1B demonstrates the two hypotheses of the construction of the bla $_{\mathrm{NDM}-1}$ into Acinetobacter species:

- The first hypothesis is a homologous recombination process between, on the one hand, a composite transposon made of two copies of ISAba125 and carrying the aphA6 gene, and, 
Figure 1. Diversity of genetic structures surrounding the bla ${ }_{\mathrm{NDM}-1}$ gene (facing page). (A) Genetic analysis comparison of the structures identified in Acinetobacter baumannii and other Gram-negative rods: Tn125 (Genbank accession number JN872329) from A. baumannii NRZ [19], pKP-NDM (Genbank accession number JN157804) from Klebsiella pneumoniae Kp7 [48], pNDM-GUE (Genbank accession number JQ364967) from Escherichia coli GUE [17] and p271A (Genbank accession number JF785549) from E. coli 271 [14]. Gene name abbreviations are deduced from their corresponding proteins. Insertion sequence elements are ISAba125, ISCR21, ISKpn7, IS26, ISEC33 and ISSen4, whereas transposon is Tn5403. aadA2 and drfA17 encode aminoglycoside and trimethoprim resistance, respectively. Base pair duplications are indicated by 3 and $8 \mathrm{bp}$. The orilS of ISCR21 is indicated by a circle. The conserved bla $a_{\mathrm{NDM}-1}$ locus is indicated by vertical black lines. (B) Possible hypotheses of the $b / a_{\mathrm{NDM}-1}$ construction: hypothesis 1 : the $b / a_{\mathrm{NDM}-1}$ gene could have been constructed by a recombination event removing the sequence between the arrows, including the aphA6 gene, and giving rise to the bla $a_{\mathrm{NDM}-1}$ gene; hypothesis 2: the bla ${ }_{\mathrm{NDM}-1}$ could have been constructed by rolling-circle transposition mediated by ISCR21 into the aphA6 gene. The yellow box into the $b / a_{\mathrm{NDM}-1}$ gene indicates the shared sequence between the $b / a_{\mathrm{NDM}-1}$ and the aphA6 genes.

IRR: Inverted repeat right; IRL: Inverted repeat left; IS: Insertion sequence; ISCR: Insertion sequence common region.

on the other hand, the bla ${ }_{\mathrm{NDM}-1}$ gene, giving rise to the neoformed $b l a_{\mathrm{NDM}-1}$ gene identified in the $\operatorname{Tn} 125$;

- The construction was due to two steps of the rolling-circle transposition event, which inserted the fragment containing the $b l a_{\mathrm{NDM}-1}$ gene from the progenitor into the $a p h A 6$ gene (Figure 1B).

In light of these recent findings, a new role for $A$. baumannii may be considered. Although usually considered as a final recipient of resistance genes, $A$. baumannii may transfer them to Enterobacteriaceae, and $P$. aeruginosa after a probable acquisition from an environmental resistome (Figure 2). To date, the role of $A$. baumannii as a source of resistance genes for other clinically significant bacterial species has not been underlined. There is only one single report of the bla ${ }_{\text {OXA-23 }}$ gene outside $A$. baumannii, being identified in Proteus mirabilis, as well as another single report of acquisition of the natural cephalosporinase bla $a_{\mathrm{ADC}}$ gene in Oligella urethralis (a Gram-negative rod belonging to Moraxellaceae $[24,25]$. However, in these two cases, A. baumannii has played the role of progenitor, whereas it played the role of an intermediate reservoir for the bla ${ }_{\mathrm{NDM}}$ genes.

\section{Epidemiology of NDM-producing Acinetobacter species}

NDM-1-producing $A$. baumannii were first identified in India [26] and then in China [27]. NDM-1-producing non-baumannii Acinetobacter, including A. lwoffii, A. junii, Acinetobacter pittii, Acinetobacter haemolyticus and Acinetobacter genomospecies 10 and 15 , have been reported from nosocomial settings and environmental sources in China [28-30]. In these studies, the $b l a_{\mathrm{NDM}-1}$ gene is carried by a plasmid with a size ranging from approximately 30 to $55 \mathrm{~kb}$, which may be transferred to $E$. coli by conjugation. Although these experiments are in vitro analyses, we might hypothesize that the dissemination of the bla ${ }_{\mathrm{NDM}}$ genes from Acinetobacter species to Enterobacteriaceae could be due to the interspecies transfer of this plasmid. In contrast to what is observed in China, a report highlighting the scattered diffusion of NDM-1-producing A. baumannii in Europe demonstrated that this dissemination was neither due to a single clone nor to plasmid diffusion, but rather to a spread of different clones carrying a same transposon Tn125 [18]. Multilocus sequence typing (MLST) analysis using Pasteur's Institute scheme [31] revealed that the clones circulating in Europe belonged to sequence type (ST)1 (also named worldwide clone I) and ST25 with a probable link with the Balkans region. Recently, several A. baumannii clinical isolates possessing the bla $a_{\mathrm{NDM}-1}$ gene and belonging to ST85 according to Pasteur's Institute MLST scheme have been identified. These isolates were recovered from patients originating from North Africa, with no obvious link to the Indian subcontinent. This finding strongly suggests that a single NDM-producing $A$. baumannii clone is likely widespread in North Africa and that it may act as a secondary reservoir of spread of the NDM-1 resistance trait [32]. These genome comparison analyses indicated that several reservoirs of NDM-1-producing $A$. baumannii have been established at least in Asia (China and India), the Balkans region and North Africa.

Hitherto, only a single NDM-1 variant has been described in A. baumannii, NDM-2 [33]. This variant has been identified in a German patient (with a travel history in Egypt), from Israel and from the United Arab Emirates [33-35]. MLST analysis revealed that these isolates belong to the same clone, ST103 (or ST253 according to MLST scheme developed by Bartual and 
(A) Tn125, Acinetobacter baumannii, chromosomal, 10,099 bp, JN872329

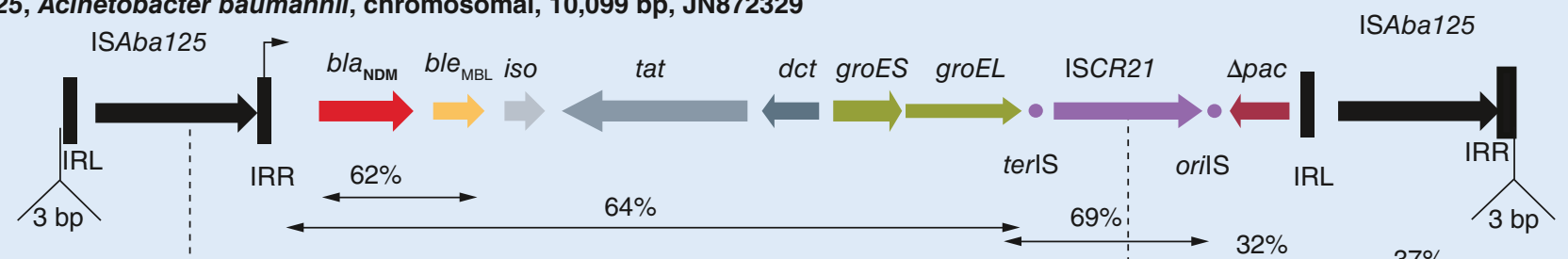

pNDM-KN, Klebsiella:pneumoniae, IncA/C, 162,746 bp, JN157804

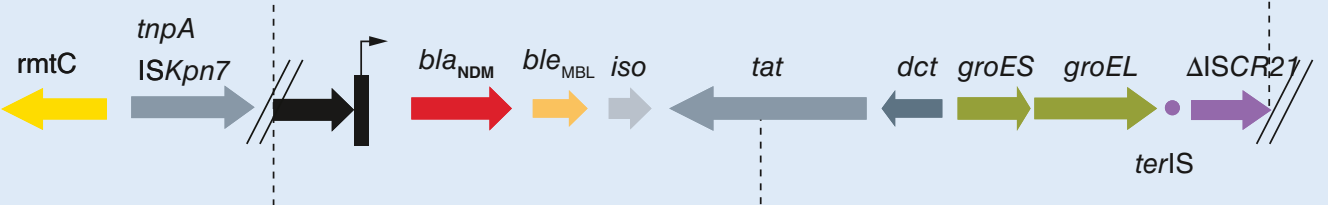

pGUE-NDM, Escherichia coli, IncFII, 87,021 bp, JQ364967

IS26

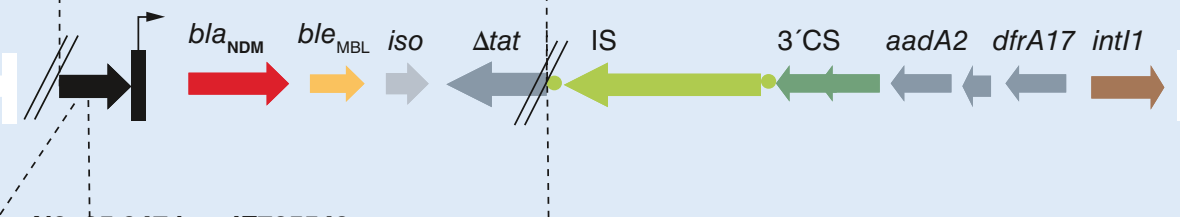

IS26

p271A, E. coli, ÍncN2, 35,947 bp, JF785549
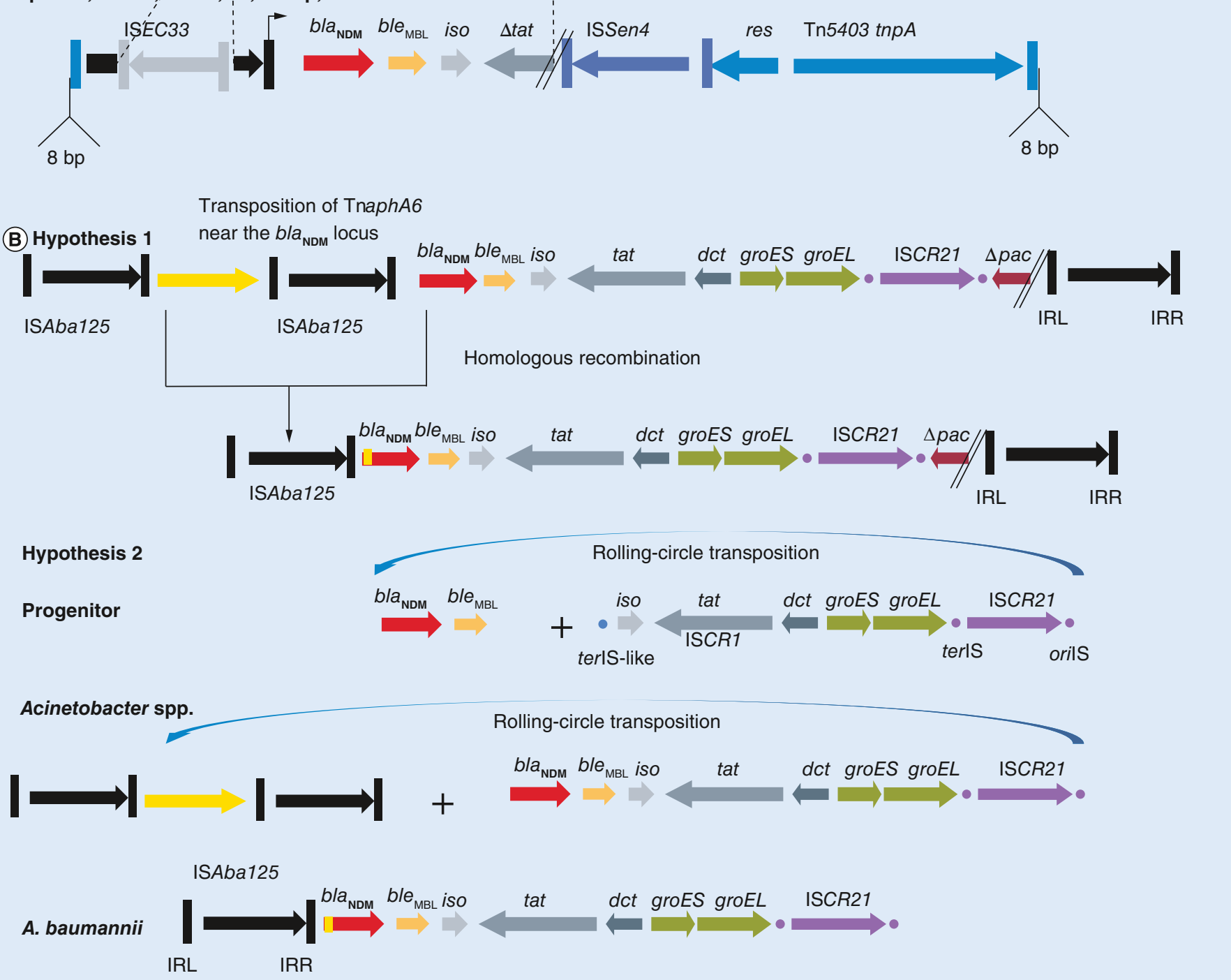


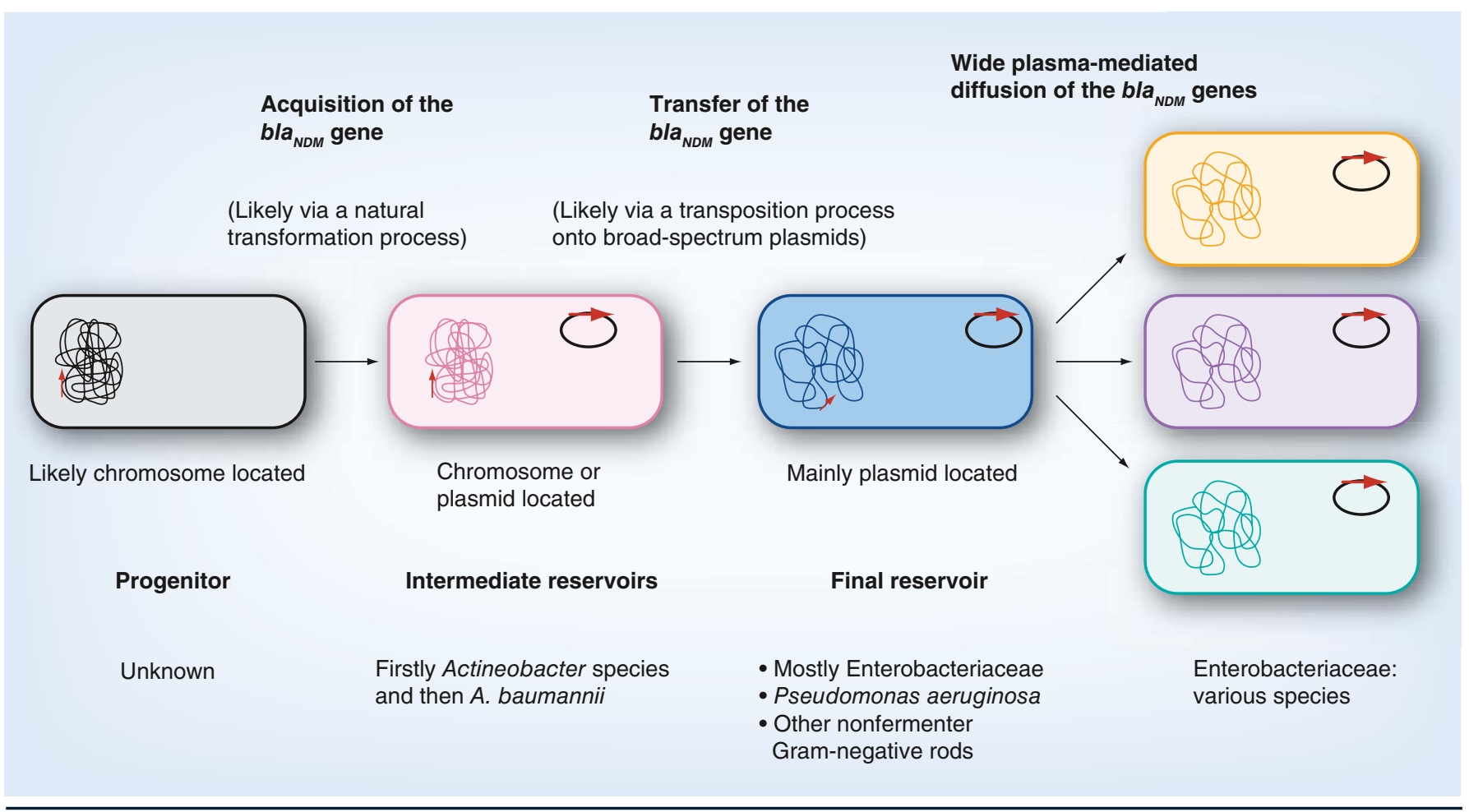

(1) Figure 2. Hypothesis of the diffusion of the bla $a_{\mathrm{NDM}}$ genes from the progenitor to clinical isolates. The different species are indicated by different colors.

colleagues [36]), corresponding to a new clone with no link to sequence types found elsewhere. In contrast to NDM-1-producing $A$. baumannii, the reservoir of the $b l a_{\mathrm{NDM}-2}$ gene seems to be present at least in the Middle East.

\section{Medical implications}

Identification of $A$. baumannii as a source of multidrug resistance is now well established. We suggest that identification of resistance genes in A. baumannii worldwide is important, not only for preventing the spread of multidrug resistance traits in A. baumannii, but also for preventing their potential transfer to Enterobacteriaceae, which are by far the most important sources of infection in humans. A. baumannii, which had been mostly considered a weak pathogen responsible for infections in immunocompromized patients, may become a Trojan horse for spreading antibiotic resistance genes in unrelated and clinically significant Gram-negative species.

More specifically, identification of NDM producers in $A$. baumannii appears to be crucial considering that these isolates have already disseminated at least in some areas (North Africa, Europe, China and India).
Another important concern is that A. baumannii is well-known to persist much longer than Enterobacteriaceae in the environment [37]. Consequently, outbreaks involving multidrug-resistant $A$. baumannii are difficult to eradicate $[1,2,38]$, as exemplified by a study demonstrating the re-emergence of the same A. baumannii clone 6 months later in a burns unit owing to environmental contamination [39]. The closure of this burns unit was necessary to eradicate the contamination [39]. Early identification is therefore very important.

\section{Future perspective}

The current diffusion of carbapenemase genes in Gram-negative rods is now a serious public health issue and, in the near future, could lead back to the preantibiotic era. The screening of multidrug-resistant $A$. baumannii and the monitoring, in particular, of the carbapenem resistance in that species has to be considered a crucial issue. The NDM saga demonstrates that the diffusion of the bla $a_{\mathrm{NDM}-1}$ gene in Enterobacteriaceae, probably originating from Acinetobacter species, shows that diffusion of resistance genes is more complex than expected. 
Financial \& competing interests disclosure

This work was partially funded by a grant from the INSERM (UMR 914) and from the European Community R-GNOSIS (HEALTH-F3-2011-282512). The authors have no other relevant affiliations or financial involvement with any organization or entity with a financial interest in or financial conflict with the subject matter or materials discussed in the manuscript apart from those disclosed.

No writing assistance was utilized in the production of this manuscript.

\section{EXECUTIVE SUMMARY}

\section{Background}

- Members of the genus Acinetobacter have the ability to rapidly develop resistance to new antibiotics.

- The main problem concerning antibiotic resistance in Acinetobacter baumannii corresponds to the rise of carbapenem resistance, which is always associated with multidrug resistance.

- A. baumannii has been considered as a final recipient of antibiotic resistance genes originating from other clinically relevant Gram-negative species.

\section{Carbapenem resistance in $A$. baumannii}

- Carbapenem-hydrolyzing Ambler class D $\beta$-lactamases are the main source of carbapenem resistance in A. baumannii.

- The acquisition of most Ambler class B and A carbapenemases has resulted from the integration of foreign DNA in A. baumannii from Enterobacteriaceae or Pseudomonas aeruginosa.

\section{The case of the bla $a_{\mathrm{NDM}}$ genes}

- The $b l a_{\mathrm{NDM}}$ genes are associated with the insertion sequence Aba125 (often truncated in Enterobacteriaceae), which plays a role in $b / a_{\mathrm{NDM}}$ expression.

- In A. baumannii, the bla $\mathrm{NDM}_{\mathrm{N}-1}$ gene is part of a 10,099-bp composite transposon made of two copies of ISAba125.

- A new role may be played by $A$. baumannii involving the acquisition of the $b / a_{\mathrm{NDM}-1}$ resistance gene and then transferring it to Enterobacteriaceae and P. aeruginosa.

\section{Epidemiology of NDM-producing Acinetobacter species}

- NDM-1-producing A. baumannii has been widely identified in India and China from nosocomial settings and environmental sources.

- Whereas the diffusion of NDM-1-producing A. baumannii in Asia seems to be due to a plasmid diffusion, in Europe, it was neither due to a single clone nor any plasmid diffusion, but rather to different clones carrying the transposon $\operatorname{Tn} 125$.

- Multilocus sequence typing analyses indicated several reservoirs of NDM-1-producing A. baumannii including Asia (mainly China and India), the Balkans region and North Africa.

\section{Medical implication}

- The identification of resistance genes in A. baumannii worldwide is important not only for preventing the spread of multidrug resistance in $A$. baumannii, but also for preventing their extension to Enterobacteriaceae.

- Outbreaks involving multidrug-resistant $A$. baumannii are known to be very difficult to eradicate owing to the ability of this bacterial species to persist in a nosocomial environment.

\section{References}

Papers of special note have been highlighted as:

- of interest

-• of considerable interest

1 Peleg AY, Seifert H, Paterson DL. Acinetobacter baumannii: emergence of a successful pathogen. Clin. Microbiol. Rev. 21(3), 538-582 (2008).

-. Important review on the biology of Acinetobacter.
2 Poirel L, Bonnin RA, Nordmann P. Genetic basis of antibiotic resistance in pathogenic Acinetobacter species. IUBMB Life 63(12), 1061-1067 (2011).

3 Fishbain J, Peleg AY. Treatment of Acinetobacter infections. Clin. Infect. Dis. 51(1), 79-84 (2010).

- Interesting review on the treatment of multidrug-resistant Acinetobacter.
4 Poirel L, Bonnin RA, Nordmann P. Genetic support and diversity of acquired extendedspectrum $\beta$-lactamases in Gram-negative rods. Infect. Genet. Evol. 12(5), 883-893 (2012).

-. Up-to-date review on the genetic of extended-spectrum $\beta$-lactamase acquisition.

5 Fournier PE, Vallenet D, Barbe $\mathrm{V}$ et al. Comparative genomics of multidrug resistance in Acinetobacter Baumannii. PLoS Genet. 2(1), e7 (2006). 
6 Iacono M, Villa L, Fortini D et al. Wholegenome pyrosequencing of an epidemic multidrug-resistant Acinetobacter baumannii strain belonging to the European clone II group. Antimicrob. Agents Chemother. 52(7), 2616-2625 (2008).

7 Poirel L, Naas T, Nordmann P. Diversity, epidemiology, and genetics of class D $\beta$-lactamases. Antimicrob. Agents Chemother. 54(1), 24-38 (2010).

8 Higgins PG, Perez-Llarena FJ, Zander E et al. OXA-235, a novel class D $\beta$-lactamase involved in resistance to carbapenems in Acinetobacter baumannii. Antimicrob. Agents Chemother. 57(5), 2121-2126 (2013).

9 Poirel L, Figueiredo S, Cattoir V, Carattoli A, Nordmann P. Acinetobacter radioresistens as a silent source of carbapenem resistance for Acinetobacter spp. Antimicrob. Agents Chemother. 52(4), 1252-1256 (2008).

10 Kumarasamy KK, Toleman MA, Walsh TR et al. Emergence of a new antibiotic resistance mechanism in India, Pakistan, and the UK: a molecular, biological, and epidemiological study. Lancet Infect. Dis. 10(9), 597-602 (2010).

First nationwide survey on the spread of the bla $a_{\mathrm{NDM}-1}$ gene.

Nordmann P, Poirel L, Walsh TR, Livermore DM. The emerging NDM carbapenemases. Trends Microbiol. 19(12), 588-595 (2011).

Yong D, Toleman MA, Giske CG et al. Characterization of a new metallo- $\beta$ lactamase gene, $b l a_{\mathrm{NDM}-1}$, and a novel erythromycin esterase gene carried on a unique genetic structure in Klebsiella pneumoniae sequence type 14 from India. Antimicrob. Agents Chemother. 53(12), 5046-5054 (2009).

- The first identification of the $b l a_{\mathrm{NDM}-1}$ gene.

13 Walsh TR, Weeks J, Livermore DM, Toleman MA. Dissemination of NDM-1 positive bacteria in the New Delhi environment and its implications for human health: an environmental point prevalence study. Lancet Infect. Dis. 11(5), 355-362 (2011).

-. Study of the spread of the $b l a_{\mathrm{NDM}-1}$ gene in the environment in New Dehli.

14 Poirel L, Bonnin RA, Nordmann P. Analysis of the resistome of a multidrug-resistant NDM-1-producing Escherichia coli strain by high-throughput genome sequencing. Antimicrob. Agents Chemother. 55(9), 4224-4229 (2011).

15 Mussi MA, Limansky AS, Viale AM. Acquisition of resistance to carbapenems in multidrug-resistant clinical strains of Acinetobacter baumannii: natural insertional inactivation of a gene encoding a member of a novel family of $\beta$-barrel outer membrane proteins. Antimicrob. Agents Chemother. 49(4), 1432-1440 (2005).

16 Lopes BS, Amyes SG. Role of ISAbal and IS $A b a 125$ in governing the expression of $b l a_{\mathrm{ADC}}$ in clinically relevant Acinetobacter baumannii strains resistant to cephalosporins. J. Med. Microbiol. 61(Pt 8), 1103-1108 (2012).

17 Bonnin RA, Poirel L, Carattoli A, Nordmann P. Characterization of an IncFII plasmid encoding NDM-1 from Escherichia coli ST131. PLoS ONE 7(4), e34752 (2012).

18 Bonnin RA, Poirel L, Naas T et al. Dissemination of New Delhi metallo- $\beta$ lactamase-1-producing Acinetobacter baumannii in Europe. Clin. Microbiol. Infect. 18(9), e362-e365 (2012).

19 Poirel L, Bonnin RA, Boulanger A et al. Tn 125-related acquisition of bla $a_{\mathrm{NDM}-}$ like genes in Acinetobacter baumannii. Antimicrob. Agents Chemother. 56(2), 1087-1089 (2012).

20 Dortet L, Nordmann P, Poirel L. Association of the emerging carbapenemase NDM-1 with a bleomycin resistance protein in Enterobacteriaceae and Acinetobacter baumannii. Antimicrob. Agents Chemother. 56(4), 1693-1697 (2012).

21 Naas T, Namdari F, Bogaerts P et al. Genetic structure associated with $b l a_{\mathrm{OXA}-18}$, encoding a clavulanic acid-inhibited extended-spectrum oxacillinase. Antimicrob. Agents Chemother. 52(11), 3898-3904 (2008).

22 Toleman MA, Bennett PM, Walsh TR. ISCR elements: novel gene-capturing systems of the 21st century? Microbiol. Mol. Biol. Rev. 70(2), 296-316 (2006)

23 Toleman MA, Spencer J, Jones L, Walsh TR. bla $_{\mathrm{NDM}-1}$ is a chimera likely constructed in Acinetobacter baumannii. Antimicrob. Agents Chemother. 56(5), 2773-2776 (2012).

24 Bonnet R, Marchandin H, Chanal C et al. Chromosome-encoded class D $\beta$-lactamase OXA-23 in Proteus mirabilis. Antimicrob. Agents Chemother. 46(6), 2004-2006 (2002).

25 Mammeri H, Poirel L, Mangeney N, Nordmann P. Chromosomal integration of a cephalosporinase gene from Acinetobacter baumannii into Oligella urethralis as a source of acquired resistance to $\beta$-lactams. Antimicrob. Agents Chemother. 47(5), 1536-1542 (2003).

26 Karthikeyan K, Thirunarayan MA, Krishnan P. Coexistence of bla ${ }_{\mathrm{OXA}-23}$ with $b l a_{\mathrm{NDM}-1}$ and $\operatorname{arm} A$ in clinical isolates of Acinetobacter baumannii from India. J. Antimicrob. Chemother. 65(10), 2253-2254 (2010).

- First description of the bla ${ }_{\mathrm{NDM}-1}$ gene in A. baumannii.
27 Chen Y, Zhou Z, Jiang Y, Yu Y. Emergence of NDM-1-producing Acinetobacter baumannii in China. J. Antimicrob. Chemother. 66(6), 1255-1259 (2011).

28 Fu Y, Du X, Ji J et al. Epidemiological characteristics and genetic structure of bla $_{\mathrm{NDM}-1}$ in non-baumannii Acinetobacter spp. in China. J. Antimicrob. Chemother. 67(9), 2114-2122 (2012).

29 Wang Y, Wu C, Zhang Q et al. Identification of New Delhi metallo- $\beta$-lactamase 1 in Acinetobacter lwoffii of food animal origin. PLoS ONE 7(5), e37152 (2012).

30 Zong Z, Zhang X. bla ${ }_{\mathrm{NDM}-1}$-carrying Acinetobacter johnsonii detected in hospital sewage. J. Antimicrob. Chemother. 68(5), 1007-1010 (2013).

31 Diancourt L, Passet V, Nemec A, Dijkshoorn $\mathrm{L}$, Brisse $\mathrm{S}$. The population structure of Acinetobacter baumannii: expanding multiresistant clones from an ancestral susceptible genetic pool. PLoS ONE 5(4), e10034 (2010).

32 Bonnin RA, Cuzon G, Poirel L, Nordmann P. Multidrug-resistant Acinetobacter baumannii clone, France. Emerg. Infect. Dis. 19(5), 822-823 (2013).

33 Kaase M, Nordmann P, Wichelhaus TA et al. NDM-2 carbapenemase in Acinetobacter baumannii from Egypt. J. Antimicrob. Chemother. 66(6), 1260-1262 (2011).

34 Espinal P, Fugazza G, Lopez Y et al. Dissemination of an NDM-2-producing Acinetobacter baumannii clone in an Israeli rehabilitation center. Antimicrob. Agents Chemother. 55(11), 5396-5398 (2011).

35 Ghazawi A, Sonnevend A, Bonnin RA et al. NDM-2 carbapenemase-producing Acinetobacter baumannii in the United Arab Emirates. Clin. Microbiol. Infect. 18(2), e34-e36 (2012).

36 Bartual SG, Seifert H, Hippler C et al. Development of a multilocus sequence typing scheme for characterization of clinical isolates of Acinetobacter baumannii. J. Clin. Microbiol. 43(9), 4382-4390 (2005).

37 Kramer A, Schwebke I, Kampf G. How long do nosocomial pathogens persist on inanimate surfaces? A systematic review. BMC Infect. Dis. 6, 130 (2006).

-. Exhaustive review on the persistence of pathogens on inanimate surfaces.

38 Adams MD, Goglin K, Molyneaux N et al. Comparative genome sequence analysis of multidrug-resistant Acinetobacter baumannii. J. Bacteriol. 190(24), 8053-8064 (2008).

39 Zanetti G, Blanc DS, Federli I et al. Importation of Acinetobacter baumannii into 
a burn unit: a recurrent outbreak of infection associated with widespread environmental contamination. Infect. Control Hosp. Epidemiol. 28(6), 723-725 (2007).

40 Krizova L, Bonnin RA, Nordmann P, Nemec A, Poirel L. Characterization of a multidrugresistant Acinetobacter baumannii strain carrying the $b l a_{\mathrm{NDM}-1}$ and $b l a_{\mathrm{OXA}-23}$ carbapenemase genes from the Czech Republic. J. Antimicrob. Chemother. 67(6), 1550-1552 (2012).

41 Gottig S, Pfeifer Y, Wichelhaus TA et al. Global spread of New Delhi metallo- $\beta$ lactamase 1. Lancet Infect. Dis. 10(12), 828-829 (2010).
42 Bogaerts P, Rezende de Castro R, Roisin S et al. Emergence of NDM-1-producing Acinetobacter baumannii in Belgium. J. Antimicrob. Chemother. 67(6), 1552-1553 (2012).

43 Nakazawa Y, Ii R, Tamura T et al. A case of NDM-1-producing Acinetobacter baumannii transferred from India to Japan. J. Infect. Chemother. 19(2), 330-332 (2013).

$44 \mathrm{Hu} \mathrm{Y}$, Zhang W, Liang $\mathrm{H}$ et al. Wholegenome sequence of a multidrug-resistant clinical isolate of Acinetobacter lwoffii. J. Bacteriol. 193(19), 5549-5550 (2011).

45 Chen Y, Cui Y, Pu F et al. Draft genome sequence of an Acinetobacter genomic species 3 strain harboring a $b l a_{\mathrm{NDM}-1}$ gene. J. Bacteriol. 194(1), 204-205 (2012).
46 Zhou Z, Guan R, Yang Y et al. Identification of New Delhi metallo- $\beta$-lactamase gene (NDM-1) from a clinical isolate of Acinetobacter junii in China. Can. J. Microbiol. 58(1), 112-115 (2012).

47 Yang J, Chen Y, Jia X et al. Dissemination and characterization of NDM-1-producing Acinetobacter pittii in an intensive care unit in China. Clin. Microbiol. Infect. 18(12), e506-e513 (2013).

48 Carattoli A, Villa L, Poirel L, Bonnin RA, Nordmann P. Evolution of IncA/C bla ${ }_{\mathrm{CMY}-2}{ }^{-}$ carrying plasmids by acquisition of the bla $_{\text {NDM-1 }}$ carbapenemase gene. Antimicrob. Agents Chemother. 56(2), 783-786 (2012). 